UPR-0818-T

hep-th/9810142

\title{
Microscopics of Rotating Black Holes: Entropy and Greybody Factors *
}

\author{
Mirjam Cvetič \\ Department of Physics and Astronomy \\ University of Pennsylvania, Philadelphia PA 19104-6396, USA
}

\begin{abstract}
We review the status of microscopic counting of rotating black hole degrees of freedom. We present two complementary approaches which both utilize the near-horizon geometry and precisely reproduce the Bekenstein Hawking entropy for near-extreme rotating black holes in $\mathrm{D}=4$ and $\mathrm{D}=5$. The first one, proposed by Strominger, is applicable for the Ramond-Ramond sector and relies on the correspondence between the near-horizon geometry and the conformal field theory on its boundary. The second one (somewhat more heuristic), employs the conformal sigma-model in the Neveu-Schwarz-NeveuSchwarz sector and accounts for the black hole microstates by counting the small scale oscillations of the dyonic string there. We also present the wave equation for the minimally coupled massive scalars for such rotating black hole backgrounds, and discuss its implication for the greybody factors. The
\end{abstract}

${ }^{*}$ Compilation of talks presented at: PASCOS'98 (March 22-27, 1998, Boston), Strings'98 (June 22-27, 1998, Santa Barbara), and 32nd International Symposium Ahrenshoop (September 1-5, 1998, Buckow, Germany). 
results are illustrated for the prototype $\mathrm{D}=5$ rotating black hole.

\section{INTRODUCTION}

One of objectives of the string theory is to shed light on physics of strong gravitational fields, such as issues of black hole information loss and related issues of black hole microscopics. In particular, important light has been shed on the latter subject of the black hole microscopics.

In a pioneering proposal [四] Sen identified the microstates of extreme (BPS-saturated) electrically charged black holes with perturbative excitations of string theory. The proposal was put on a firmer footing [2,3] by interpreting the extreme electric black hole states as oscillating modes of an underlying macroscopic (fundamental) string. However, only after the discovery of non-perturbative multi-charge black holes [4, [5] with finite BekensteinHawking entropy, attempts to establish a quantitative agreement between the microscopic and macroscopic entropy in string theory became feasible. Such solutions were originally specified by charges from the Neveu-Schwarz-Neveu-Schwarz (NS-NS) sector [5,6]. Their microscopic features were captured by string theory in curved space-time geometry of the near-horizon region which is that of an $S L(2, R) \times S U(2)$ Wess-Zumino-Witten (WZW) model [7,8]. In particular, the small-scale string oscillations [9,8] were shown to reproduce [8.10.11] the extreme black hole entropy directly from the near-horizon geometry.

These developments were overshadowed by the advent of D-branes - nonperturbative objects in string theory with Ramond-Ramond (R-R) charges [12]. (Black holes with NS-NS charges can be mapped, by duality symmetry, onto black holes with R-R charges which have the same space-time metric and thus Bekenstein-Hawking entropy.) The higher-dimensional interpretation of these black holes in terms of intersecting D-branes lead to a counting of black hole quantum states that agrees precisely with the Bekenstein-Hawking entropy for both the extreme [13] and near-extreme [14,15] static, as well as rotating [16, 17] black holes. 
Recently, the implications of the near-horizon geometry for microscopics of black holes was resurrected. Strominger [18] (see also [19,20]) has given an alternative derivation of the Bekenstein-Hawking entropy, by again employing the near-horizon geometry: the central observation is that, when embedded in a higher dimensional space, the near-horizon geometry contains Bañados-Teitelboim-Zanelli black hole (BTZ) [21] space-time which is locally that of the three-dimensional anti de Sitter space-time $\left(A d S_{3}\right)$, whose quantum states are determined by a two-dimensional conformal field theory (CFT) at the asymptotic boundary [22. The counting of states in this CFT is then used to reproduce the Bekenstein-Hawking entropy.

In the following we review the microscopics of rotating black holes within the two complementary approaches, both utilizing the black hole near-horizon region. The first one employs the $\mathrm{AdS}_{3} / \mathrm{CFT}$ correspondence [23]; the method proposed by Strominger is applied to near-extreme rotating black holes (with rotation fully included) [24,25], thus generalizing the results for static near-extreme black holes in $\mathrm{D}=5$ [18] and $\mathrm{D}=4$ [26], respectively.

The second approach provides a counting of microstates in the NS-NS sector by relying on the correspondence (matching) between the solitonic and fundamental string states [8, [10,[1],27]. The microstates are counted directly as string states at the horizon and not at the asymptotic boundary. Recent analysis [27] generalizes the earlier [8,10,11] counting of microstates for BPS-saturated black-holes to the case of non-extreme black holes. The string sigma-model representing a non-extreme black hole with NS-NS charges is still equiva-

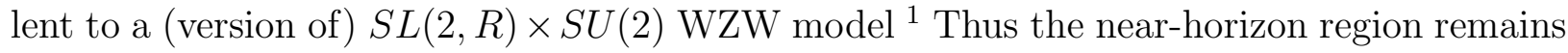
to be effectively described by the free fundamental string whose tension is again rescaled by the magnetic charges. However, now the black-hole microstates are identified not only with the left-moving, but also right-moving superconformal string oscillations, which can be of the same order of magnitude.

\footnotetext{
${ }^{1}$ A connection of BTZ [21] space-time to $S L(2)$ WZW Lagrangian was explored in [28,29].
} 
The results are reviewed for the prototype example of the generating solution for general $\mathrm{D}=5$ rotating black holes of toroidally compactified string theory. (The discussion for $\mathrm{D}=4$ rotating black holes is analogous.) The rest of the contribution is organized in the following way. In Section II the black hole parameterization is given and the Bekenstein-Hawking entropy is displayed. In Section III the near-horizon geometry of $\mathrm{D}=6$ rotating dyonic string (obtained by lifting the $\mathrm{D}=5$ black hole solution) in the decoupling limit is discussed and the counting following [18 is presented. The minimally coupled massive scalar wave equation and its implication for the greybody factors is discussed in Section IV. In Section V the conformal sigma model, specified by the near-horizon target space of the $\mathrm{D}=6$ rotating string in the NS-NS sector is addressed and counting of microstates in this sector is presented.

\section{FIVE-DIMENSIONAL ROTATING BLACK HOLE}

The metric for the classical solution for the prototype black hole, which correspond to the generating solution for the most general $\mathrm{D}=5$ rotating black hole in $N=4$ or $N=8$ supergravity in five dimensions was given explicitly in [30]. It is specified by $M$, two angular momenta $J_{L, R}$, and three independent $U(1)$ charges $Q_{i}$. It is convenient to represent these physical parameters in the parametric form:

$$
\begin{aligned}
M & =m \sum_{i=0}^{2} \cosh 2 \delta_{i}, \\
Q_{i} & =m \sinh 2 \delta_{i} \quad ; \quad i=0,1,2, \\
J_{L, R} & =m\left(l_{1} \mp l_{2}\right)\left(\prod_{i=0}^{2} \cosh \delta_{i} \pm \prod_{i=0}^{2} \sinh \delta_{i}\right) .
\end{aligned}
$$

We work in Planck units where the gravitational coupling constant in five dimensions is $G_{5}=\frac{\pi}{4}$.

The Bekenstein-Hawking entropy is of the form [31:

$$
\begin{aligned}
S \equiv \frac{A_{5}}{4 G_{5}} & =2 \pi m\left[\left(\prod_{i=0}^{2} \cosh \delta_{i}+\prod_{i=0}^{2} \sinh \delta_{i}\right)\left(r_{+}+r_{-}\right)+\right. \\
& \left.+\left(\prod_{i=0}^{2} \cosh \delta_{i}-\prod_{i=0}^{2} \sinh \delta_{i}\right)\left(r_{+}-r_{-}\right)\right]
\end{aligned}
$$


where

$$
r_{ \pm}=\frac{1}{2}\left[\sqrt{2 m-\left(l_{1}-l_{2}\right)^{2}} \pm \sqrt{2 m-\left(l_{1}+l_{2}\right)^{2}}\right]
$$

is the chosen value of the radial coordinate at the outer and inner horizon, respectively. The two charges $Q_{1}$ and $Q_{2}$ appear symmetrically and can be interpreted as those of a fundamental string (FS) and a NS5-brane and $Q_{0}$ a momentum along the common direction. By duality $Q_{1}$ and $Q_{2}$ charges can be interpreted as those of D1- and D5-brane, respectively. D-branes are accessible to a weakly coupled microscopic description addressed in the following Section, while the NS-NS sector solution has a conformal sigma-model interpetation addressed in Section V.

\section{D=6 ROTATING STRING IN DECOUPLING LIMIT}

The above rotating black hole can be lifted to $D=6$ and has an interpretation as a dyonic rotating black string. Its Einstein-frame metric was given explicitly in [24]. Its internal structure (and that of the corresponding black hole) can be described accurately by a field theory that couples weakly to the surrounding space. A precise definition of the decoupling limit is given by taking [23]:

$$
l_{s} \rightarrow 0 ; r, m, l_{1,2} \rightarrow 0 \quad ; \delta_{1,2} \rightarrow \infty
$$

where the string length $l_{s}=\sqrt{\alpha^{\prime}}$, so that:

$$
r l_{s}^{-2} ; m l_{s}^{-4} ; l_{1,2} l_{s}^{-2} ; Q_{1,2} l_{s}^{-2}=m l_{s}^{-2} \sinh 2 \delta_{1,2}
$$

remain fixed. The decoupling limit is a near horizon approximation, because $r \rightarrow 0$, while the "dilute gas" conditions $\delta_{1,2} \rightarrow \infty$ imply that the black hole is necessarily near extremal 40 .

The metric of $\mathrm{D}=6$ rotating dyonic string simplifies dramatically in the limit specified by eq. 6. The near horizon geometry is:

$$
d s_{6}^{2}=\frac{r^{2}}{f_{D} \lambda^{2}}\left[-\left(1-\frac{2 m f_{D}}{r^{2}}\right) d \tilde{t}^{2}+d \tilde{y}^{2}\right]+\frac{\lambda^{2} r^{2}}{\left(r^{2}+l_{1}^{2}\right)\left(r^{2}+l_{2}^{2}\right)-2 m r^{2}} d r^{2}-
$$




$$
\begin{aligned}
& -2\left(l_{2} \cos ^{2} \theta d \psi+l_{1} \sin ^{2} \theta d \phi\right) d \tilde{t}-2\left(l_{1} \cos ^{2} \theta d \psi+l_{2} \sin ^{2} \theta d \phi\right) d \tilde{y}+ \\
& +\lambda^{2}\left(d \theta^{2}+\sin ^{2} \theta d \phi^{2}+\cos ^{2} \theta d \psi^{2}\right),
\end{aligned}
$$

where

$$
f_{D}^{-1}=1+\frac{l_{1}^{2} \cos ^{2} \theta}{r^{2}}+\frac{l_{2}^{2} \cos ^{2} \theta}{r^{2}},
$$

and

$$
\begin{aligned}
& \tilde{t}=\cosh \delta_{0} t-\sinh \delta_{0} y, \\
& \tilde{y}=\cosh \delta_{0} y-\sinh \delta_{0} t,
\end{aligned}
$$

are the boosted coordinates along the string direction. The characteristic length scale $\lambda$ is defined as $\lambda \equiv\left(Q_{1} Q_{2}\right)^{\frac{1}{4}}$.

Introducing the shift in the angular variables:

$$
\begin{aligned}
& \tilde{\psi}=\psi-\lambda^{-2}\left(l_{2} d \tilde{t}+l_{1} \tilde{y}\right), \\
& \tilde{\phi}=\phi-\lambda^{-2}\left(l_{1} d \tilde{t}+l_{2} \tilde{y}\right),
\end{aligned}
$$

the metric becomes:

$$
\begin{aligned}
d s_{6}^{2} & =-\frac{\left(r^{2}+l_{1}^{2}\right)\left(r^{2}+l_{2}^{2}\right)-2 m r^{2}}{\lambda^{2} r^{2}} d \tilde{t}^{2}+\frac{r^{2}}{\lambda^{2}}\left(d \tilde{y}-\frac{l_{1} l_{2}}{r^{2}} d \tilde{t}\right)^{2}+ \\
& +\frac{\lambda^{2} r^{2}}{\left(r^{2}+l_{1}^{2}\right)\left(r^{2}+l_{2}^{2}\right)-2 m r^{2}} d r^{2}+\lambda^{2}\left[d \theta^{2}+\sin ^{2} \theta d \tilde{\phi}^{2}+\cos ^{2} \theta d \tilde{\psi}^{2}\right]
\end{aligned}
$$

In this form it is apparent that the geometry is a direct product of two three-dimensional spaces. The angular space is a sphere $S^{3}$ with radius $\lambda$, and the geometry with coordinates $(\tilde{t}, \tilde{y}, r)$ is a BTZ black hole in an effective $2+1$ dimensional theory with cosmological constant $\Lambda=-\lambda^{2}$. The metric can be written in the standard BTZ form [21] with the effective three dimensional mass $M_{3}$ and angular momentum $J_{3}$ :

$$
\begin{aligned}
M_{3} & =\frac{R_{y}^{2}}{\lambda^{4}}\left[\left(2 m-l_{1}^{2}-l_{2}^{2}\right) \cosh 2 \delta_{0}+2 l_{1} l_{2} \sinh 2 \delta_{0}\right], \\
8 G_{3} J_{3} & =\frac{R_{y}^{2}}{\lambda^{3}}\left[\left(2 m-l_{1}^{2}-l_{2}^{2}\right) \sinh 2 \delta_{0}+2 l_{1} l_{2} \cosh 2 \delta_{0}\right] .
\end{aligned}
$$


The radius of the compact dimension is denoted by $R_{y}$.

Thus, the local space-time remains that of $A d S_{3} \times S^{3}$ (the same as for the static dyonic string) with the role of angular momenta encoded in the global space-time structure!

\section{A. Counting of States}

We now summarize the counting of the black hole microstates, following [18]. The effective gravitational coupling in three dimensions, $G_{3}$, can be related to the gravitational coupling in five dimensions, $G_{5}\left(=\frac{\pi}{4}\right)$, by comparing two different dimensional reductions from six dimensions, as in [18,26. It is:

$$
\frac{1}{G_{3}}=\frac{1}{G_{5}} \frac{A_{3}}{2 \pi R_{y}},
$$

where $A_{3}=2 \pi^{2} \lambda^{3}$ is the area of the $S^{3}$. This result is independent of the rotational parameters because the effective cosmological constant depends only on the charges of the branes.

The isometry group of the asymptotic $A d S_{3}$ induces a conformal field theory on the boundary at the conformal infinity of the BTZ black hole. Its central charge is given in terms of the cosmological constant [22]:

$$
c=\frac{3 \lambda}{2 G_{3}}=6 \frac{Q_{1} Q_{2}}{R_{y}} .
$$

Note that the central charge is also independent of angular momentum. This suggests that the rotating black holes can be interpreted as states in the same conformal field theory that describes the nonrotating black holes.

The relation between the symmetry generators of the induced conformal symmetry, and the effective mass and angular momentum are:

$$
\begin{aligned}
M_{3} & =\frac{8 G_{3}}{\lambda}\left(L_{0}+\bar{L}_{0}\right), \\
J_{3} & =L_{0}-\bar{L}_{0},
\end{aligned}
$$


where the eigenvalues of the operators $L_{0}$ and $\bar{L}_{0}$ are the conformal dimensions $N_{L}$ and $N_{R}$, respectively. Then Cardy's formula [32] for the statistical entropy:

$$
S=2 \pi\left(\sqrt{\frac{c N_{L}}{6}}+\sqrt{\frac{c N_{R}}{6}}\right),
$$

gives the microscopic entropy:

$$
\begin{aligned}
S_{\text {stat }} & =\frac{\pi}{4 G_{3}}\left[\sqrt{\lambda\left(\lambda M_{3}+8 G_{3} J_{3}\right)}+\sqrt{\lambda\left(\lambda M_{3}-8 G_{3} J_{3}\right)}\right] \\
& =\pi \sqrt{Q_{1} Q_{2}}\left[\left(r_{+}+r_{-}\right) e^{\delta_{0}}+\left(r_{+}-r_{-}\right) e^{-\delta_{0}}\right],
\end{aligned}
$$

which precisely matches macroscopic entropy eq. 0 in the limit $\delta_{1,2} \gg 1$. Thus the microscopic entropy eq. 22 precisely reproduces in the decoupling limit eq. 6 where the microscopic calculation applies. The range of parameters that are considered here is as general as the previous D-brane results [16:17].

\section{BLACK HOLE PERTURBATIONS}

The isometry group $S O(2,2) \simeq S L(2, R)_{L} \times S L(2, R)_{R}$ of $A d S_{3}$ can be exploited in several ways. The computation of the entropy relies on the fact that the BTZ black hole is asymptotically $A d S_{3}$, so that a conformal field theory is induced at the boundary at infinity. However, the BTZ geometry is in fact locally $A d S_{3}$. This has important consequences for the spectrum of black hole perturbations [33 39], and for the dynamics encoded in the greybody factors. Here we discuss some of these issues with an emphasis on the effects of rotation.

An important consequence of the local $A d S_{3} \times S^{3}$ form of the metric is that the spectrum of black hole perturbations is organized into multiplets of the superconformal algebra. This

allows a complete classification of all perturbations, as carried out in [35]. The spectrum of perturbations follows from local properties of $A d S_{3}$, and so it is identical for the entire class of black holes considered here.

The perturbations are naturally interpreted as test fields that interact with the black hole background. The wave function of the perturbations then gives the greybody factor, expressing the form factor of the Hawking radiation as a function of particle quantum 
numbers, such as energy and spin, and the black hole parameters 40 47. The greybody factors provide a semiclassical testing ground for dynamical properties. In the special case of minimally coupled scalars in the S-wave they agree precisely with calculations in string theory 48]. The microscopic processes responsible for other modes can be modelled in terms of an effective string theory with dynamics that reproduces the black hole greybody factors qualitatively 41,42.

\section{A. Minimally Coupled Massive Scalar Wave Equation}

For the sake of concreteness we discuss the case of a minimally coupled massive scalar field. By using the original coordinates $t, y$ and the radial variable $x$ defined as

$$
x \equiv \frac{r^{2}-\frac{1}{2}\left(r_{+}^{2}+r_{-}^{2}\right)}{r_{+}^{2}-r_{-}^{2}},
$$

the near-horizon metric eq. 8 is of the form:

$$
\begin{aligned}
d s_{6}^{2} & =\lambda^{-2}\left[-\left(x-\frac{1}{2}\right)\left(r_{+} d \tilde{t}-r_{-} d \tilde{y}\right)^{2}+\left(x+\frac{1}{2}\right)\left(r_{+} d \tilde{y}-r_{-} d \tilde{t}\right)^{2}\right]+ \\
& +\lambda^{2}\left[\frac{1}{4 x^{2}-1} d x^{2}+d \theta^{2}+\cos ^{2} \theta d \tilde{\psi}^{2}+\sin ^{2} \theta d \tilde{\phi}^{2}\right]
\end{aligned}
$$

where $(\tilde{t}, \tilde{y})$ and $(\tilde{\psi}, \tilde{\phi})$ are defined in eqs. 1011 and eqs. 12 13, respectively. Inserting the following Ansatz for the wave function to:

$$
\Phi=\Phi_{0}(r) \chi(\theta) e^{-i \omega_{R}(t+y)-i \omega_{L}(t-y)+i m_{R}(\phi+\psi)+i m_{L}(\phi-\psi)}
$$

into the Klein-Gordon equation:

\footnotetext{
2 The fact that there is a separation of variables for this rotating background with the massive minimally coupled scalars is nontrivial. (The same observation was made for massless minimally coupled scalars in the five dimensional black hole geometry [43].) It indicates that there may be additional conserved Killing-Stackel-type tensors, analogous to the case of Kerr black holes in four dimensions. (For some discussion and references see [49,50].)
} 


$$
\frac{1}{\sqrt{-g}} \partial_{\mu}\left(\sqrt{-g} g^{\mu \nu} \partial_{\nu} \Phi\right)=\mu^{2}
$$

one finds the following form of the radial wave equation [24]:

$$
\begin{aligned}
& {\left[\frac{\partial}{\partial x}\left(4 x^{2}-1\right) \frac{\partial}{\partial x}+\frac{1}{x-\frac{1}{2}}\left(\frac{\beta^{R} \omega_{R}+\beta^{L} \omega_{L}-m_{R} \beta_{H} \Omega^{R}-m_{L} \beta_{H} \Omega^{L}}{2 \pi}\right)^{2}\right.} \\
& \left.-\frac{1}{x+\frac{1}{2}}\left(\frac{\beta^{R} \omega_{R}-\beta^{L} \omega_{L}-m_{R} \beta_{H} \Omega^{R}+m_{L} \beta_{H} \Omega^{L}}{2 \pi}\right)^{2}\right] \Phi_{0}=\left(\Lambda+\lambda^{2} \mu^{2}\right) \Phi_{0} .
\end{aligned}
$$

Here the eigenvalues of the angular Laplacian:

$$
\hat{\Lambda}=-\frac{1}{\sin 2 \theta} \frac{\partial}{\partial \theta} \sin 2 \theta \frac{\partial}{\partial \theta}-\frac{1}{\sin ^{2} \theta} \frac{\partial^{2}}{\partial \phi^{2}}-\frac{1}{\cos ^{2} \theta} \frac{\partial^{2}}{\partial \psi^{2}},
$$

are denoted $\Lambda$ and take the form $\Lambda=l(l+2)$ where $l=0,1, \cdots$. In eq. 27

$$
\beta^{L, R}=\frac{2 \pi \lambda^{2} e^{\mp \delta_{0}}}{\sqrt{2 m-\left(l_{1} \mp l_{2}\right)^{2}}}, \quad \beta_{H} \Omega^{L, R}=\frac{2 \pi\left(l_{1} \mp l_{2}\right)}{\sqrt{2 m-\left(l_{1} \mp l_{2}\right)^{2}}} .
$$

$\beta^{L, R}$ have an interpretation of potentials conjugate to the left- and right-moving energy of the 2d-CFT (with $\beta_{H}=\frac{1}{2}\left(\beta_{L}+\beta_{R}\right)$ corresponding to the inverse of the Hawking temperature); $\beta_{H} \Omega^{L, R}$ are conjugate to the two angular momenta $J_{L, R}$, respectively.

The wave equation eq. 27 agrees with the near horizon limit of the general one given in [43], except that there the Ansatz for the wave function did not allow dependence on the compact coordinate $y$ [3. The present generalization gives an even more symmetric result.

It follows immediately from the form of the wave equation eq. 27 that its solutions depend on $m_{L, R}$ and $\beta_{H} \Omega^{L, R}$ only through the prescriptions:

$$
\beta^{L, R} \omega_{L, R} \rightarrow \beta^{L, R} \omega_{L, R}-m_{L, R} \beta_{H} \Omega^{L, R}
$$

\footnotetext{
${ }^{3}$ An equation that applies in the general nonextremal case and includes the dependence on the compact coordinate $y$ can be obtained from the master equation given in 43, by exploiting boost invariance in the $y$ dimension. In 43 it was also observed that the near-horizon region of the radial wave equation respects the $S L(2, R)_{L} \times S L(2, R)_{R}$ symmetry leading to a conjecture that this symmetry has a 2-d CFT origin. However, a connection of this CFT to the $A d S_{3}$ was not recognized, since in the five-dimensional context the dependence on the $y$ direction was suppressed.
} 
This rule gives the exact wave functions in the rotating background, when the nonrotating ones are known. (It turns out to be valid for all fields in the near-horizon region, without regard to the details of their couplings.) Note also that a mass $\mu$ is included in the KleinGordon equation, eq. 26, and its effect can be absorbed in the conformal dimension. The solution to the near horizon wave equation is a hypergeometric function found in 42,51.52 (present notation is used in 443).

\section{CONFORMAL SIGMA MODEL FOR ROTATING BLACK HOLES}

We now turn to the study of black hole microscopics in the NS-NS sector. We demonstrate [27] that for a large NS5-brane and fundmental string the black hole lifted to $\mathrm{D}=6$ is described by a marginal integrable perturbation of the $S L(2, R) \times S U(2)$ WZW model generated by the left- and right-moving Cartan chiral $S U(2)$ currents whose perturbation parameters are proportional to the two angular momentum components.

In NS-NS sector the $\mathrm{D}=6$ rotating dyonic string solution is described by the three NS-NS charges: the NS5-brane charge $Q_{1}$, the fundamental string $(\mathrm{FS})$ charge $Q_{2}$ and the string momentum $Q_{0}$. Its Einstein frame metric is the same as that specified by R-R-charges. The string sigma-model corresponding to this background has a complicated form, however it simplifies significantly in the near-horizon region. In this limit, it takes the following form 27]:

$$
\begin{aligned}
(L)_{\delta_{1,2} \gg 1} & =Q_{2}^{-1}\left[\left(r^{2} f_{D}^{-1}-m\right) \partial U \bar{\partial} V+\frac{1}{2} m e^{2 \delta_{0}} \partial U \bar{\partial} U+\frac{1}{2} m e^{-2 \delta_{0}} \partial V \bar{\partial} V\right]+ \\
& -\sqrt{\frac{Q_{1}}{Q_{2}}}\left[\left(l_{1}-l_{2}\right) e^{\delta_{0}} \partial U \bar{J}_{3}+\left(l_{1}+l_{2}\right) e^{-\delta_{0}} J_{3} \bar{\partial} V\right] \\
& +Q_{1}\left[\frac{r^{2} \partial r \bar{\partial} r}{\left(r^{2}-r_{+}^{2}\right)\left(r^{2}-r_{-}^{2}\right)}+L_{S U(2)}\right],
\end{aligned}
$$

\footnotetext{
${ }^{4}$ For the sake of making the notations of Sections III, and V compatible, the convention here differs from that of 27] in the following way: $\left(Q_{0}, Q_{1}, Q_{2}\right)_{\text {here }}=(\tilde{Q}, P, Q)_{\text {there }},(J, \bar{J})_{\text {here }}=2(J, \bar{J})_{\text {there }}$, and $e_{\text {here }}^{\delta_{0}}=e^{-\delta_{\tilde{Q}}}$ there .
} 
with the constant dilaton $e^{2 \Phi}=\frac{Q_{1}}{Q_{2}}$. Here the light-cone string coordinates are defined as: $(U, V)=\mp t+y$, while

$$
L_{S U(2)}=\partial \theta \bar{\partial} \theta+\sin ^{2} \theta \partial \varphi \bar{\partial} \varphi+\cos ^{2} \theta \partial \psi \bar{\partial} \psi
$$

is the $S U(2)$ WZW Lagrangian with the $S U(2)$ chiral Cartan currents:

$$
J_{3}=\sin ^{2} \theta \partial \varphi+\cos ^{2} \theta \partial \psi, \quad \bar{J}_{3}=-\sin ^{2} \theta \bar{\partial} \psi+\cos ^{2} \theta \bar{\partial} \varphi
$$

It is apparent that the Lagrangian eq. 31 can be interpreted as a chiral marginal perurbation of a $S L(2, R) \times S U(2)$ WZW model by the $S U(2)$ Cartan currents eq. 33. This can be confirmed by noting that after the coordinate transformation [24]

$$
\begin{aligned}
& \varphi \rightarrow \varphi-\frac{1}{2}\left(Q_{1} Q_{2}\right)^{-\frac{1}{2}}\left[-\left(l_{1}-l_{2}\right) e^{\delta_{0}} U+\left(l_{1}+l_{2}\right) e^{-\delta_{0}} V\right], \\
& \psi \rightarrow \psi-\frac{1}{2}\left(Q_{1} Q_{2}\right)^{-\frac{1}{2}}\left[\left(l_{1}-l_{2}\right) e^{\delta_{0}} U+\left(l_{1}+l_{2}\right) e^{-\delta_{0}} V\right],
\end{aligned}
$$

the Lagrangian eq. 31 assumes the form:

$$
\begin{aligned}
(L)_{\delta_{1,2} \gg 1} & =Q_{2}^{-1}\left[\left(r^{2}-\frac{1}{2}\left(r_{+}^{2}+r_{-}^{2}\right)\right) \partial U \bar{\partial} V+\frac{1}{4}\left(r_{+}+r_{-}\right)^{2} e^{2 \delta_{0}} \partial U \bar{\partial} U\right. \\
& \left.+\frac{1}{4}\left(r_{+}-r_{-}\right)^{2} e^{-2 \delta_{0}} \partial V \bar{\partial} V\right]+Q_{1}\left[\frac{r^{2} \partial r \bar{\partial} r}{\left(r^{2}-r_{+}^{2}\right)\left(r^{2}-r_{-}^{2}\right)}+L_{S U(2)}\right]
\end{aligned}
$$

which is precisely the $S L(2, R) \times S U(2)$ WZW Lagrangian at level $\kappa=Q_{1}$ ?!

\section{A. Statistical entropy}

The aim will be to employ the structure of the $S L(2, R) \times S U(2)$ model eq. 35 to give a microscopic interpretation of the Bekenstein-Hawking entropy eq. Tin the limit of large $Q_{1}, Q_{2}$ charges.

\footnotetext{
5 Note that transformations: $\cosh \frac{z}{2}=\sqrt{\frac{r^{2}-r_{-}^{2}}{r_{+}^{2}-r_{-}^{2}}}, u=\frac{1}{2 \sqrt{Q_{1} Q_{2}}}\left(r_{+}+r_{-}\right) e^{\delta_{0}} U$ and $v=\frac{1}{2 \sqrt{Q_{1} Q_{2}}}\left(r_{+}-r_{-}\right) e^{-\delta_{0}} V$ render $L_{S L(2)}$ part of the Lagrangian eq. 35 in the canonical form: $L_{S L(2)}=\kappa(2 \cosh z \partial u \bar{\partial} v+\partial u \bar{\partial} u+\partial v \bar{\partial} v+\partial z \bar{\partial} z)$ where $\kappa=Q_{1}$.
} 
The main idea (originated in [8], further refined in [10,11] and generalized to include the right-moving oscillations in [27]) is that in the large charge limit the $S L(2, R) \times S U(2) \mathrm{WZW}$ model describes a fundamental string with a tension rescaled by the NS5-brane charge $Q_{1}$, and whose relevant marginal deformations are only those of the four transverse directions to the NS5-brane. Since the charges are large, the conformal sigma-model eq. 35 is weakly coupled, so one is to count string states in nearly-flat space. This implies that the central $c$ charge of the "free" effective superstring that accounts for the bosonic and fermionic excitations in the four transverse directions is

$$
c=4\left(1+\frac{1}{2}\right)=6 .
$$

In order to relate the oscillation numbers $N_{L, R}$ of the fundamental string and thus its entropy to the global parameters of the black hole one is to use the "matching conditions" analogous to the ones in [2, 3, 11]. Below we describe the counting procedure of the small scale oscillations for the WZW Lagrangian eq. 35.

Let us combine the $z$-direction (related to $r$ ) of $L_{S L(2)}$ Largangian, and three angular coordinates of $(\theta, \varphi, \psi)$ of $L_{S U(2)}$ Lagrangian into four transverse coordinates $x^{i}$. Since the WZW level $\kappa=Q_{1}$ is large, the interactions in WZW theory are suppressed by $1 / \kappa$, and thus the count of perturbations should be essentially the same as in the theory of free fields $x^{i}$. The only difference compared to the flat space case is the presence of the factor $\kappa$ in front of the kinetic term of $x^{i}$ coordinates (cf. eq. 35). The sigma-model which represents the perturbed version of the model eq. 35 by left- as well as right-moving perturbations can be written as

$$
\begin{aligned}
L & =Q_{2}^{-1}\left[F(x) \partial U \bar{\partial} V+K_{0} \partial U \bar{\partial} U+M_{0} \partial V \bar{\partial} V\right. \\
& \left.+\mathcal{A}_{i}(x, U) \partial U \bar{\partial} x^{i}+\tilde{\mathcal{A}}_{i}(x, V) \partial x^{i} \bar{\partial} V\right]+Q_{1} h_{i j}(x) \partial x^{i} \bar{\partial} x^{j} .
\end{aligned}
$$

Here $(U, V)$ correspond to the string light-cone coordinates $(U, V)=\mp t+y$ of the effective fundamental string. Since the level $\kappa=Q_{1}$ is large, we may assume that $h_{i j}$ is approximately flat. As a result, the perturbations $\mathcal{A}_{i}(x, U)$ and $\tilde{\mathcal{A}}_{i}(x, V)$ are marginal to the leading order 
in their strength. Indeed, integrating out $U$ "freezes" $V$ and thus makes $\tilde{\mathcal{A}}_{i}$ marginal and vice versa for $\mathcal{A}_{i}$. In general, there is also a constraint on their $x$-dependence, and the solution which is relevant in the large charge limit is

$$
\mathcal{A}_{i}(x, U) \sim a_{i}(U), \quad \tilde{\mathcal{A}}_{i}(x, V) \rightarrow b_{i}(V) .
$$

Integrating out the four transverse fields $x^{i}$ in the large $\kappa$ limit we find that $K_{0}$ and $M_{0}$ in (37) are replaced by

$$
K(U)=K_{0}-\left(Q_{1} Q_{2}\right)^{-1} a_{i}^{2}(U), \quad M(V)=M_{0}-\left(Q_{1} Q_{2}\right)^{-1} b_{i}^{2}(V) .
$$

(In general, there is also a $\mathcal{A} \tilde{\mathcal{A}}$-correction to the $\partial U \bar{\partial} V$ term.)

The analogue of the level matching condition for the free fundamental string [2, 3] which relates the oscillation level numbers $N_{L}$ and $N_{R}$ to the background charges is as follows: the coefficients of both $\partial U \bar{\partial} U$ and $\partial V \bar{\partial} V$ terms should vanish on average to allow matching onto a fundamental string source, i.e.

$$
\bar{K}=0, \quad \bar{M}=0, \quad \bar{f} \equiv \frac{1}{2 \pi R} \int_{0}^{2 \pi R} d y f(y),
$$

where $R$ is the radius of a compact direction along which the string is wound. Then, to the leading order in $1 / \kappa$,

$$
\overline{a_{i}^{2}}=Q_{1} Q_{2} K_{0}=N_{L}, \quad \overline{b_{i}^{2}}=Q_{1} Q_{2} M_{0}=N_{R},
$$

where $N_{L}$ and $N_{R}$ are interpreted as the left- and the right-moving string oscillation numbers. (The proportionality constant between $N_{L, R}$ and $\left(K_{0}, M_{0}\right)$ depends on the tension of the fundamental string and is equal to 1 in units used (i.e., $\left.G_{5}=\frac{\pi}{4}\right)$.)

Note that for the Lagrangian eq. 35 the relationship eq. 41 implies:

$$
\begin{aligned}
& N_{L}=Q_{1} Q_{2} K_{0}=\frac{1}{4} Q_{1} Q_{2}\left(r_{+}+r_{-}\right)^{2} e^{2 \delta_{0}}, \\
& N_{R}=Q_{1} Q_{2} M_{0}=\frac{1}{4} Q_{1} Q_{2}\left(r_{+}-r_{-}\right)^{2} e^{-2 \delta_{0}} .
\end{aligned}
$$

Inserting the value of the central charge eq. 36 and the oscillator numbers $N_{L, R}$ eq. 42 into Cardy's entropy formula eq. 36 one obtains: 


$$
S_{\text {stat }}=\pi \sqrt{Q_{1} Q_{2}}\left[\left(r_{+}+r_{-}\right) e^{\delta_{0}}+\left(r_{+}-r_{-}\right) e^{-\delta_{0}}\right]
$$

which is indeed in agreement with that of eq. 14 in the limit if large charges.

\section{ACKNOWLEDGMENTS}

I would like to thank F. Larsen and A. Tseytlin for collaboration on the work presented in this contribution. The work is supported in part by DOE grant DOE-FG02-95ER40893. 


\section{REFERENCES}

[1] A. Sen. Extremal black holes and elementary string states. Mod. Phys. Lett. A, 10:20812094, 1995. hep-th/9504147.

[2] C.G. Callan, J.M. Maldacena and A.W. Peet. Extremal black holes as fundamental strings. Nucl. Phys. B, 475:645-678, 1996. hep-th/9510134.

[3] A. Dabholkar, J.P. Gauntlett, J.A. Harvey and D. Waldram. Strings as solitons and black holes as strings. Nucl. Phys. B, 474:85-121, 1996. hep-th/9511053.

[4] R. Kallosh, A. Linde, T. Ortin, A. Peet, and A. Van Proeyen. Supersymmetry as a cosmic censor, Phys. Rev. D, 46:5278-5302, 1992. hep-th/9205027.

[5] M. Cvetič and D. Youm. Dyonic BPS-saturated black holes of heterotic string theory on a six-torus. Phys. Rev. D, 53:584-588, 1996. hep-th/9507090.

[6] M. Cvetič and A.A. Tseytlin. General class of BPS saturated dyonic black holes as exact superstring solutions. Phys. Lett. B, 366:95-103, 1996. hep-th/9510097.

[7] D. Lowe and A. Strominger. Exact four-dimensional dyonic black holes and BertottiRobinson space-times in string theory. Phys. Rev. Lett., 73:1468-1471, 1994. hepth/9403186.

[8] M. Cvetič and A. Tseytlin. Solitonic strings and BPS saturated dyonic black holes. Phys. Rev. D, 53:5619-5633, 1996. hep-th/9512031; Erratum-ibid. 55:3907, 1997.

[9] F. Larsen and F. Wilczek. Internal structure of black holes. Phys. Lett. B, 375:37-42, 1996. hep-th/9511064.

[10] A.A. Tseytlin. Extreme dyonic black holes in string theory. Mod.Phys.Lett.A, 11:689714, 1996. hep-th/9601177.

[11] A.A. Tseytlin. Extremal black hole entropy from string sigma model. Nucl. Phys., B477:431-448, 1996. hep-th/9605091. 
[12] J. Polchinski. Dirichlet branes and Ramond-Ramond charges. Phys. Rev. Lett., 75:47244727, 1995. hep-th/9510017.

[13] A. Strominger and C. Vafa. Microscopic origin of the Bekenstein-Hawking entropy. Phys. Lett. B, 379:99-104, 1996. hep-th/9601029.

[14] C.G. Callan and J.M. Maldacena. D-brane approach to black hole quantum mechanics. Nucl. Phys. B, 472:591-610, 1996. hep-th/9602043.

[15] G. Horowitz and A. Strominger. Counting states of near-extremal black holes. Phys. Rev. Lett., 77:2368-2371, 1996. hep-th/9602051.

[16] J.C. Breckenridge, R.C. Myers, A.W. Peet and C. Vafa. D-branes and spinning black holes. Phys. Lett. B, 391:93-98, 1997. hep-th/9602065.

[17] J.C. Breckenridge, D.A. Lowe, R.C. Myers, A.W. Peet, A. Strominger and C. Vafa. Macroscopic and microscopic entropy of near-extremal spinning black holes. Phys. Lett. $B, 381: 423-426,1996$. hep-th/9603078.

[18] A. Strominger. Black hole entropy from near horizon microstates. hep-th/9712251.

[19] D. Birmingham, I. Sachs and S. Sen. Entropy of three-dimensional black holes in string theory. hep-th/9801019.

[20] K. Sfetsos and A. Skenderis. Microscopic derivation of the Bekenstein-Hawking entropy for non-extremal black holes. Nucl. Phys. B, 517:179-204, 1998. hep-th/9711138.

[21] M. Banados, Teitelboim, and J. Zanelli. The black hole in three-dimensional space-time. Phys. Rev. Lett., 69:1849-1851, 1992. hep-th/9204099.

[22] J.D. Brown and M. Henneaux. Central charges in the canonical realization of asymptotic symmetries: An example from three-dimensional gravity. Comm. Math. Phys., 104:207226, 1986.

[23] J. Maldacena. The large N limit of superconformal field theories and supergravity. hep- 
th/9711200.

[24] M. Cvetič and F. Larsen. Near horizon geometry of rotating black holes in five dimensions. hep-th/9805097.

[25] M. Cvetič and F. Larsen. Microstates and near-horizon geometry for rotating black holes in four dimensions. hep-th/9805146.

[26] V. Balasubramanian and F. Larsen. Near horizon geometry and black holes in four dimensions. hep-th/9802198.

[27] M. Cvetič and A. Tseytlin. Sigma model of near-extreme rotating black holes and their microstates. hep-th/9806141.

[28] G.T. Horowitz and D.L. Welch. Exact three-dimensional black holes in string theory. Phys. Rev. Lett. 71:328-331, 1993. hep-th/9302126.

[29] N. Kaloper. Miens of the three-dimensional black hole. Phys. Rev. D, 48:2598-2605,1993 hep-th/9303007.

[30] M. Cvetič and D. Youm. General rotating five-dimensional black holes of toroidally compactified heterotic string. Nucl. Phys. B, 476:118-132, 1996. hep-th/9603100.

[31] M. Cvetič and D. Youm. Entropy of non-extreme charged rotating black holes in string theory. Phys. Rev. D, 54:2612-2620, 1996. hep-th/9603147.

[32] J. L. Cardy. Conformal invariance and statistical mechanics. In E.Brézin and J. ZinnJustin, editors, Fields, Strings, and Critical Phenomena. Les Houches, 1988.

[33] J. Maldacena and A. Strominger. $A d S_{3}$ black holes and a stringy exclusion principle. hep-th/9804085.

[34] E. Martinec. Matrix models of AdS gravity. hep-th/9804111.

[35] S. Deger, A. Kaya, E. Sezgin, and P. Sundell. Spectrum of D =6, N=4B supergravity 
on $A d S_{3} \times S_{3}$. hep-th/9804166.

[36] F. Larsen. The perturbation spectrum of black holes in $\mathrm{N}=8$ supergravity. hepth/9805208

[37] J de Boer. Six-dimensional supergravity on $S^{3} \times A d S_{3}$ and 2-d conformal field theory. hep-th/9806104.

[38] A. Giveon, D. Kutasov and N. Seiberg. Comments on string theory on $\operatorname{AdS}(3)$. hepth/9806194.

[39] E. Martinec. Conformal field theory, geometry and entropy. hep-th/9809021.

[40] J. Maldacena and A. Strominger. Black hole greybody factors and D-brane spectroscopy. Phys. Rev. D, 55:861-870, 1996. hep-th/9609026.

[41] C. G. Callan, S. S. Gubser, I. R. Klebanov, and A. A. Tseytlin. Absorption of fixed scalars and the D-brane approach to black holes. Nucl. Phys. B, 489:65-94, 1997. hepth/9610172.

[42] J. Maldacena and A. Strominger. Universal low-energy dynamics for rotating black holes. Phys. Rev. D, 56:4975-4983, 1997. hep-th/9702015.

[43] M. Cvetič and F. Larsen. General rotating black holes in string theory: Greybody factors and event horizons. Phys. Rev. D, 56:4994-5007, 1997. hep-th/9705192.

[44] M. Cvetič and F. Larsen. Greybody factors for rotating black holes in four-dimensions. Nucl. Phys. B, 506:107-120, 1997. hep-th/9705192.

[45] S. Gubser. Absorption of photons and fermions by black holes in four-dimensions. Phys. Rev. D, 56:7854-7868, 1997. hep-th/9706100.

[46] K. Hosomichi. Fermion emission from five-dimensional black holes. hep-th/9711072.

[47] M. Cvetič and F. Larsen. Greybody factors for black holes in four-dimensions: Particles 
with spin. hep-th/9712118.

[48] S. Das and S. Mathur. Comparing decay rates for black holes and D-branes. Nucl. Phys. $B, 478: 561-576,1996$. hep-th/9606185.

[49] G. Gibbons, R. Rietdijk, and J. van Holten. SUSY in the sky. Nucl. Phys. B, 404:42-64, 1993. hep-th/9303112.

[50] M. Cvetič and F. Larsen. Black hole horizons and the thermodynamics of strings. Nucl. Phys. B (Proc. Suppl.), 62:443-456, 1998. hep-th/9706071.

[51] S. Gubser. Can the effective string see higher partial waves? Phys. Rev. D, 56:4984-4993, 1997. hep-th/9704195.

[52] S. Mathur. Absorption of angular momentum by black holes and d-branes. Nucl. Phys. B, 514:204-226, 1998. hep-th/9704156. 